\title{
THE IMPACT OF THE RELIABILITY OF TELEINFORMATION SYSTEMS ON THE QUALITY OF TRANSMITTED INFORMATION
}

\author{
WPLYW NIEZAWODNOŚCI SYSTEMÓW \\ TELEINFORMATYCZNYCH NA JAKOŚĆ \\ PRZESYLANYCH INFORMACJI
}

\author{
Marek Stawowy, Zbigniew Kasprzyk, Andrzej Szmigiel \\ Politechnika Warszawska \\ e-mail:mst@wt.pw.edu.pl
}

\begin{abstract}
The work describes the impact the reliability of the information quality $I Q$ for information and communication systems. One of the components of IQ is the reliability properties such as relativity, accuracy, timeliness, completeness, consistency, adequacy, accessibility, credibility, congruence. Each of these components of IQ is independent and to properly estimate the value of IQ, use one of the methods of modeling uncertainty. In this article, we used a hybrid method that has been developed jointly by one of the authors. This method is based on the mathematical theory of evidence know as Dempstera-Shafera (DS) theory and serial links of dependent hybrid named IQ (hyb).
\end{abstract}

Keywords: modeling, reliability, information quality, information and communications technology

Streszczenie: W pracy opisano wptyw niezawodności na jakoś informacji IQ (ang. - Information Quality) przesytanych w systemach teleinformatycznych. Jedna ze sktadowych IQ jest niezawodność obok takich właściwości jak relatywność, dokładność, aktualność, kompletność, spójność, odpowiedniość, dostepność, wiarygodność, przystawalność. Każda z tych składowych IQ jest niezależna $i$ aby poprawnie oszacować wartość IQ należy użyć jednej z metod modelowanie niepewności. $W$ artykule tym zastosowano metode hybrydowa opracowana wspólnie przez jednego z autorów artykutu. Metoda ta opiera się na ewidencji matematycznej znanej jako teoria Dempstera-Shafera (DS) oraz szeregowych powiazaniach zależnych nazwanych hybrydowymi IQ(hyb).

Stowa kluczowe: modelowanie, niezawodność, jakość informacji, teleinformatyka 
The impact of the reliability of teleinformation systems on the quality...

Wpływ niezawodności systemów teleinformatycznych na jakość przesyłanych...

\section{THE IMPACT OF THE RELIABILITY OF TELEINFORMATION SYSTEMS ON THE QUALITY OF TRANSMITTED INFORMATION}

\section{Introduction}

This article describes the impact of the reliability on information quality IQ in information and communication systems. According to [2] reliability is one of the components of IQ among other properties such as: relativity, accuracy, timeliness, completeness, consistency, adequacy, accessibility, credibility, congruence. Each of these components of IQ are independent and in order to properly estimate the value of IQ, one of the methods of modeling uncertainty has to be used. In this article, we used a hybrid method that has been developed jointly by one of the authors and presented in [8]. This method is based on the mathematical theory of evidence know as Dempster-Shafer (DS) [1,5] theory and serial links of dependent [4] named hybrid IQ (hyb) [8]. This article exhibits a way of determining information quality by using a hybrid method. The model and the calculations were limited to the impact of reliability of devices and the correctness of data transmission for this IQ. By determining the IQ a comparative assessment of communication and information systems e.g. in video surveillance (like the one's presented in $[3,6]$ ) can be easily performed.

\section{IQ model for a chosen fragment of the information and communication network.}

IQ model for a chosen fragment of the information and communication network is presented and circled in figure 1.

The IQ dependency on several properties of the system is independent and parallel. Yet in some cases it can occur, that it should depend on the consecutive stages of determining these parallel properties. That explains why the hybrid method was chosen, as it renders serial calculations and parallel dependencies (both dependent and independent) possible.

In the case of the structure of the chosen for modeling fragment of the network it is possible to determine one main hypothesis $h$, which factor will be the IQ factor (hyb), but also three intermediate hypotheses for each of the applied devices.

Hybrid IQ model based on hybrid modeling will be described with intermediate hypotheses:

h1 - error-free data transmission by a chosen fragment of network,

$\mathrm{h} 2$ - failure of data transmission due to software defect or external interference,

h3 - failure of data transmission due to device or cable damage. 


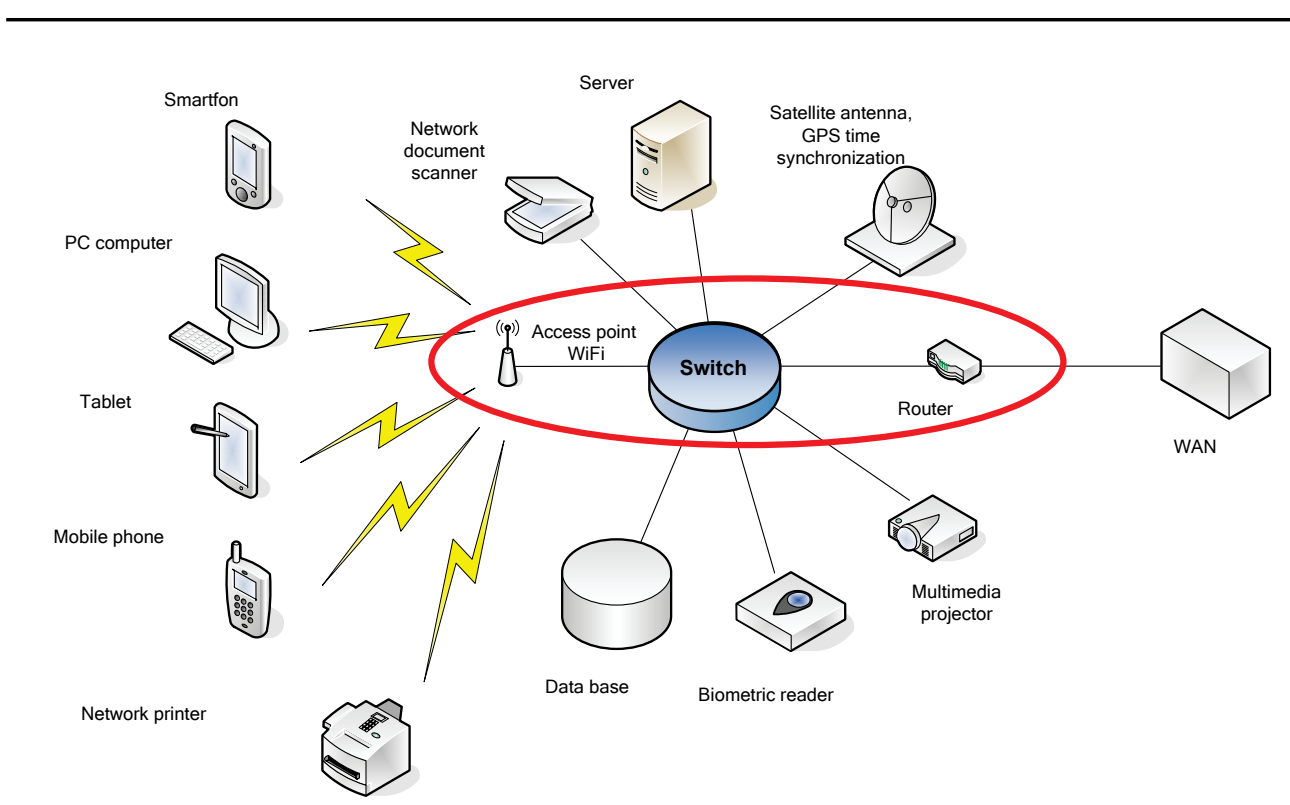

Fig. 1. An exemplary information and communication network with an outlined fragment applied for modeling IQ. Prepared by the author.

Observations for each of the intermediate hypotheses are as follows:

For h1 hypothesis:

e1.1 - error-free data transfer by access point,

e 1.2 - error-free data transfer by the switch,

e 1.3 - error-free data transfer by the router.

For h2 hypothesis:

e2.1 - failure of data transmission or AP software defect,

e2.2 - failure of data transmission or switch software defect,

e2.3 - failure of data transmission or router software defect.

For h3 hypothesis:

e3.1 - AP damage,

e3.2 - cable damage,

e3.3 - switch damage,

e3.4 - router damage.

The observation coefficient values are presented in table 1 . These values are not empirical and serve only the purpose of demonstrating the method's potential.

Table 1: The observation coefficient values for intermediate hypotheses.

\begin{tabular}{|c|c|c|}
\hline $\mathrm{h} 1$ & $\mathrm{~h} 2$ & $\mathrm{~h} 3$ \\
\hline $\mathrm{e} 1.1=0,997$ & $\mathrm{e} 2.1=0,1 * 10^{-3}$ & $\mathrm{e} 3.1=0,1 * 10^{-3}$ \\
\hline $\mathrm{e} 1.2=0,999$ & $\mathrm{e} 2.2=0,5 * 10^{-4}$ & $\mathrm{e} 3.2=0,15^{*} 10^{-5}$ \\
\hline $\mathrm{e} 1.3=0,9992$ & $\mathrm{e} 2.3=0,3 * 10^{-3}$ & $\mathrm{e} 3.3=0,5 * 10^{-4}$ \\
\hline $\mathrm{x}$ & $\mathrm{x}$ & $\mathrm{e} 3.4=0,2 * 10^{-3}$ \\
\hline
\end{tabular}


The impact of the reliability of teleinformation systems on the quality...

Wptyw niezawodności systemów teleinformatycznych na jakość przesyłanych...

Fig. 2 presents a graph of dependent model of intermediate hypotheses of independent model.

Fig. 3 shows graphs of respective dependent models for each intermediate hypothesis.

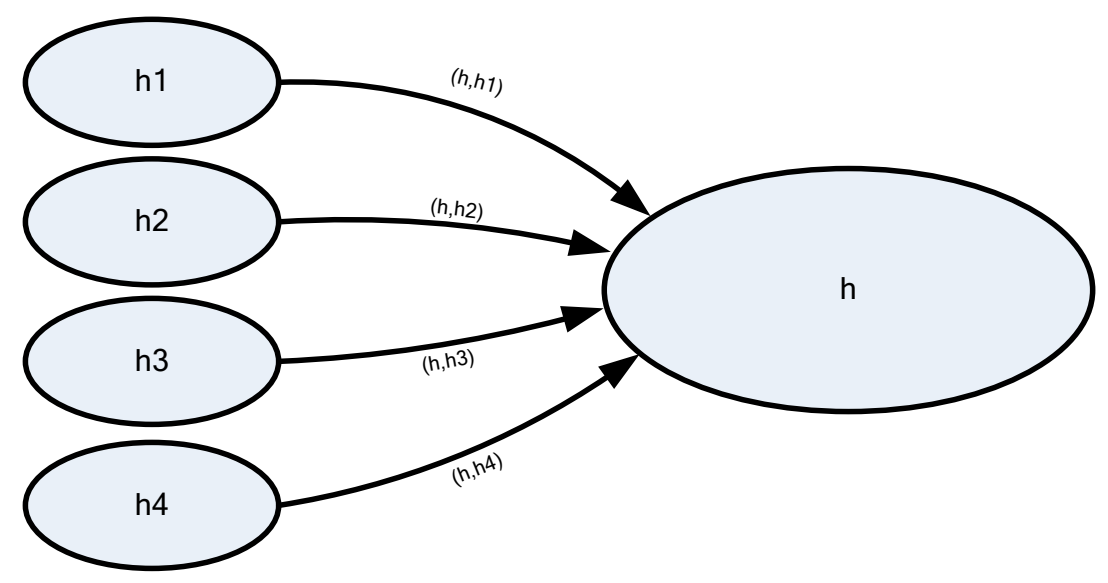

Fig. 2.Graph of independent intermediate hypotheses. Prepared by author.

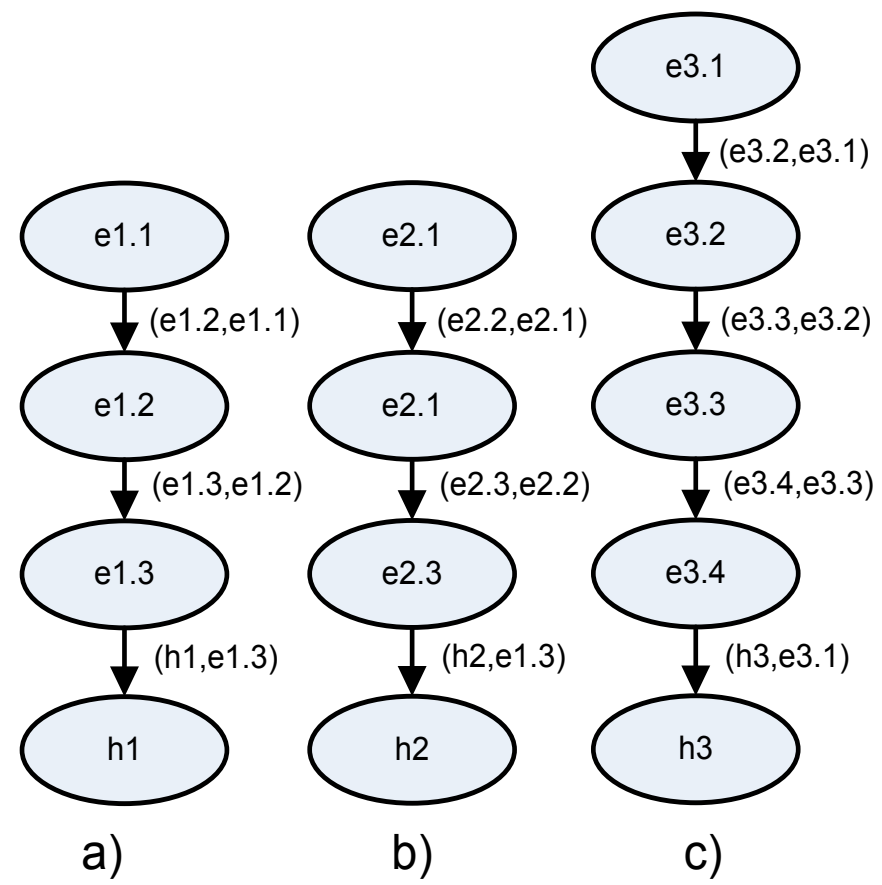

Fig. 3. Graphs of independent observations of intermediate hypotheses a) h1, b) h2, c) h3. Prepared by author 
The intermediate hypotheses coefficients were determined employing scalar product as described in $[4,8]$. Calculations resulted with coefficients presented in table 2 .

Table 2: Values of determined intermediate hypotheses.

\begin{tabular}{|c|c|c|}
\hline $\mathrm{h} 1$ & $\mathrm{~h} 2$ & $\mathrm{~h} 3$ \\
\hline 0,988034976 & $0,15^{*} 10^{-11}$ & $0,15^{*} 10^{-17}$ \\
\hline
\end{tabular}

The theory of mathematical evidence developed by Dempster and Shafer (DS) facilitates synthesizing the properties of various independent sources. $[1,5,7,8]$. By employing this theory it is possible to determine final hypothesis coefficient $h$ as presented hereunder.

Three observations of effects were defined on the basis of observing causes and intermediate hypotheses:

Ze1 - correct data, (h1),

Ze2 - data did not reach due to software defect or external interference (h2),

Ze3 - data did not reach due to hardware failure (h3).

Tables 3, 4 and 5 illustrate consecutive stages of results observations analysis.

$$
\begin{gathered}
\Theta=\{\mathrm{h} 1, \mathrm{~h} 2, \mathrm{~h} 3\} \\
\mathrm{m}_{1}(\Theta)=1 \\
\mathrm{~m}_{2}(\{\mathrm{~h} 1\})=0,988034976 \\
\mathrm{~m}_{2}(\Theta)=\mathrm{m}_{1}(\Theta)-\mathrm{m}_{2}(\{\mathrm{~h} 1\})
\end{gathered}
$$

Table 3: Observation Ze1.

\begin{tabular}{|l|l|l|}
\hline & $\mathrm{m}_{2}(\{\mathrm{~h} 1\})$ & $\mathrm{m}_{2}(\Theta)$ \\
\hline $\mathrm{m}_{1}(\Theta)$ & $\mathrm{m}_{3}(\{\mathrm{~h} 1\})$ & $\mathrm{m}_{3}(\Theta)$ \\
\hline \multicolumn{3}{|c|}{$\mathrm{m}_{4}(\{\mathrm{~h} 2\})=0,15 * 10^{-11}$} \\
$\mathrm{~m}_{4}(\Theta)=\mathrm{m}_{1}(\Theta)-\mathrm{m}_{4}(\{\mathrm{~h} 2\})$
\end{tabular}

Table 4: Observation Ze2.

\begin{tabular}{|l|l|l|}
\hline & $\mathrm{m}_{4}(\{\mathrm{~h} 2\})$ & $\mathrm{m}_{4}(\Theta)$ \\
\hline $\mathrm{m}_{3}(\{\mathrm{~h} 1\})$ & $\mathrm{m}_{5}(\{\varnothing\})$ & $\mathrm{m}_{5}(\{\mathrm{~h} 1\})$ \\
\hline $\mathrm{m}_{3}(\Theta)$ & $\mathrm{m}_{5}(\{\mathrm{~h} 2\})$ & $\mathrm{m}_{5}(\Theta)$ \\
\hline
\end{tabular}

$$
\begin{gathered}
\mathrm{m}_{6}(\{\mathrm{~h} 3\})=0,15^{*} 10^{-17} \\
\mathrm{~m}_{6}(\Theta)=\mathrm{m}_{1}(\Theta)-\mathrm{m}_{6}(\{\mathrm{~h} 3\})
\end{gathered}
$$

Table 5: Observation Ze3.

\begin{tabular}{|l|l|l|}
\hline & $\mathrm{m}_{6}(\{\mathrm{~h} 3\})$ & $\mathrm{m}_{6}(\Theta)$ \\
\hline $\mathrm{m}_{5}(\{\varnothing\})$ & $\mathrm{m}_{7}(\{\varnothing\})$ & $\mathrm{m}_{7}(\{\varnothing\})$ \\
\hline $\mathrm{m}_{5}(\{\mathrm{~h} 2\})$ & $\mathrm{m}_{7}(\{\mathrm{~h} 2\})$ & $\mathrm{m}_{7}(\{\mathrm{~h} 2\})$ \\
\hline $\mathrm{m}_{5}(\{\mathrm{~h} 1\})$ & $\mathrm{m}_{7}(\{\varnothing\})$ & $\mathrm{m}_{7}(\{\mathrm{~h} 1\})$ \\
\hline $\mathrm{m}_{5}(\Theta)$ & $\mathrm{m}_{7}(\{\mathrm{~h} 3\})$ & $\mathrm{m}_{7}(\Theta)$ \\
\hline
\end{tabular}

$$
\operatorname{Bel}(\mathrm{h} 1)=\mathrm{m}_{7}(\mathrm{~h} 1)
$$


The impact of the reliability of teleinformation systems on the quality...

Wpływ niezawodności systemów teleinformatycznych na jakość przesyłanych...

On the basis of deliberations in [7,8] it can be assumed that Bel(h1) equals IQ coefficient of the presented model. Namely IQ(hyb) $=$ Bel(h1) and after placing exemplary values from table 2 it amounts to 0,988034975999982 .

\section{Simulation and results.}

The computer simulation employed normal distribution to exhibit the impact of reliability on IQ. 10000 simulation steps were executed with rejection of values outside interval $<0,1>$. Simulation software was written by one of the authors for the needs of this article.

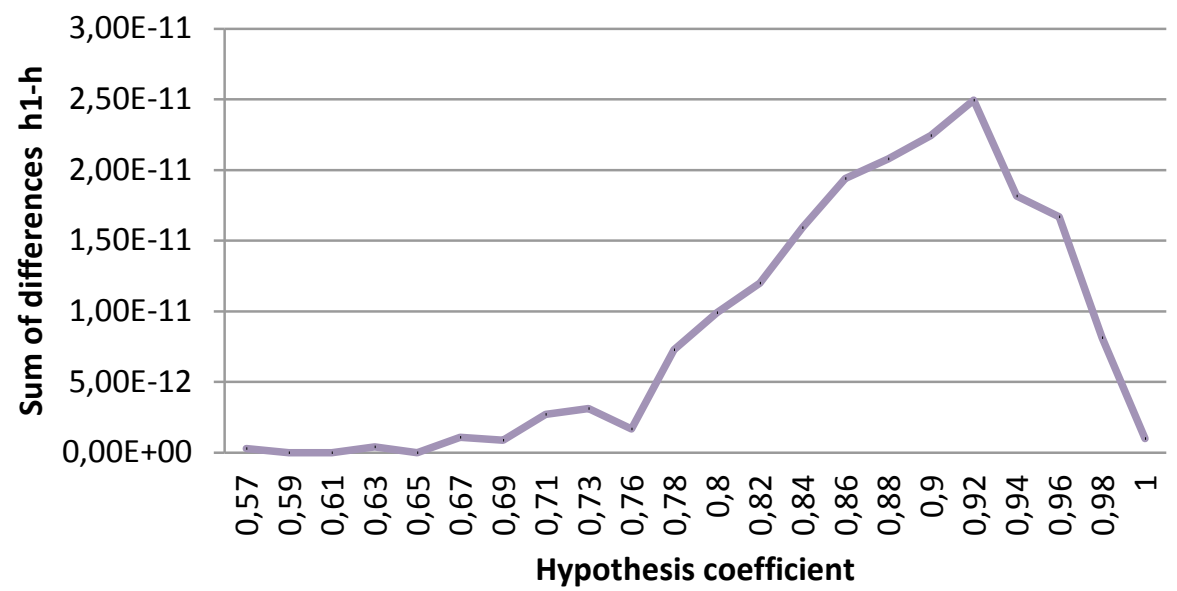

Fig. 4. The result of the simulation - sum of differences $h 1-h$.

Prepared by the author.

Fig. 4 presents a graph of the sum of differences h1-h in the consecutive stages of simulation. The line of the graph exhibits how intermediate observations $\mathrm{h} 2$ and h3, namely those connected with reliability, considerably influence the hypothesis coefficient value $h$.

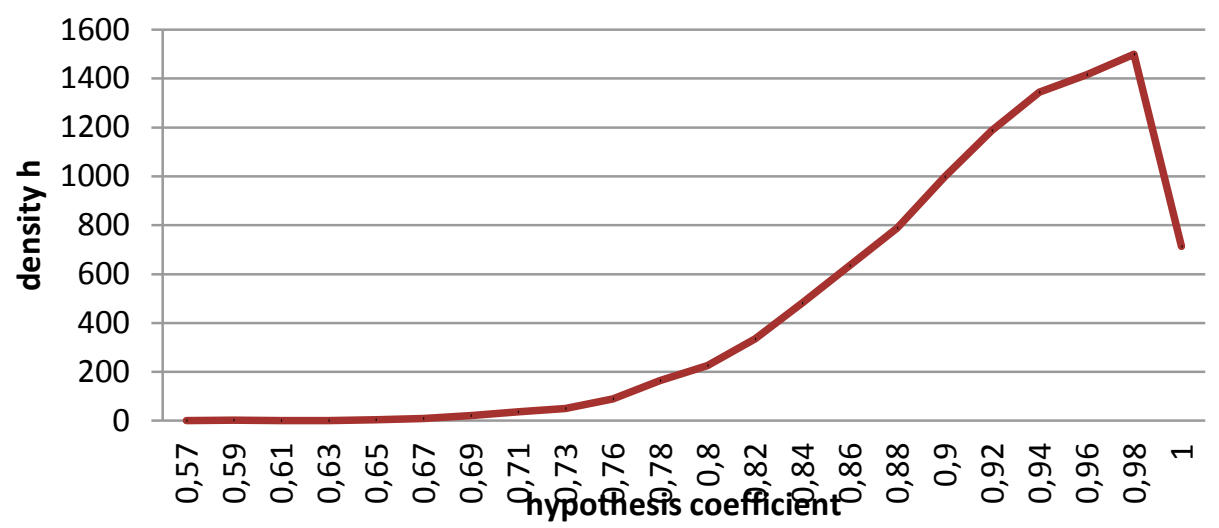

Fig. 5. Simulation result - density h. Prepared by the author. 
Fig. 5 illustrates the results of assessment simulation IQ(hyb). The average value deviates insignificantly from the value calculated in the previous chapter because the simulation denied values bigger than 1 .

\section{Conclusions}

This article presented and assigned but also simulated the impact of reliability on the IQ of a chosen information and communication system. The method presented in the article, adopting hybrid modeling to determine IQ, facilitates easy and quick calculations of IQ on the basis of dependent and independent properties but also serial and parallel properties. It is at the same time easy to implement as a computer program due to the repeatability of the majority of calculations. In the case presented in this article, the method enables assessment of the impact of various properties on IQ, particularly the influence of reliability. In the subsequent stage the calculations should be determined by time.

\section{References}

[1] Dempster A. P. Upper and lower probabilities inducted by a multi-valued mapping. The Annals of Mathematical Statistics 38, 1967, 325-339. USA.

[2] Olaisen, J. Information quality factors and the cognitive authority of electronic information. Wormell, I. (Ed.), Information Quality: definitions and dimensions, London 1990: Taylor Graham.

[3] Lubkowski, P. and Laskowski, D. (2014) The selected issues of reliable identification of object in transport systems using video monitoring services. In the monograph Telematics - support for transport, given as the monographic publishing series - „Communications in Computer and Information Science”, Vol. 471. Springer-Verlag, Berlin Heidelberg, pp. 59-68.

[4] Pieczynski, W. Unsupervised Dempster-Shafer fusion of dependent sensors, Proc. 4th IEEE Southwest Symp. Image Analysis and Interpretation, 2000.

[5] Shafer, G. A mathematical theory of evidence. Princeton University Press. Princeton 1976.

[6] Siergiejczyk M., Paś J., Rosiński A.: Evaluation of safety of highway CCTV system's maintenance process. The monograph „Telematics - support for transport”, editors: Mikulski J., given as the monographic publishing series „Communications in Computer and Information Science”, Vol. 471. SpringerVerlag, Berlin Heidelberg 2014. pp. 69-79.

[7] Stawowy M., Comparison of Uncertainty Models of Impact of Teleinformation Devices Reliability on Information Quality, Proceedings of the European Safety and Reliability Conference ESREL 2014", editors Nowakowski T., Młyńczak M., Jodejko-Pietruczuk A. \& WerbińskaWojciechowska S. The publisher: CRC Press/Balkema, 2015. Pages 2329-2333. 
The impact of the reliability of teleinformation systems on the quality...

Wplyw niezawodności systemów teleinformatycznych na jakość przesytanych...

[8] Stawowy M., Dziula P., Comparison of uncertainty multilayer models of impact of teleinformation devices reliability on Information Quality, Proceedings of the European Safety and Reliability Conference ESREL 2015, editors Luca Podofillini,Bruno Sudret,Bozidar Stojadinovic,Enrico Zio,Wolfgang Kröger CRC Press/Balkema, 2015. pp 2685-2691.

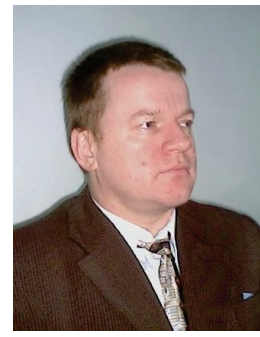

Marek Stawowy - Assistant Professor, Department of Transport, Warsaw University of Technology. Research interests: Computer image analysis, information quality, modeling uncertainties quality information. Author of dozens of scientific-technical article (Share $80 \%$ ).

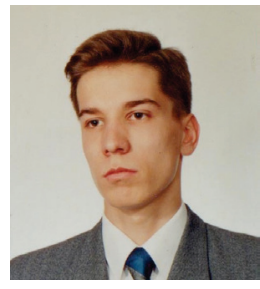

Zbigniew Kasprzyk - Assistant Professor, Department of Transport, Warsaw University of Technology. Research interests: modeling of toll systems, modeling of the functions of transport telematic equipments, analysis of operating systems, reliability analysis of data communication networks. Author of dozens of scientific-technical article (Share 10\%).

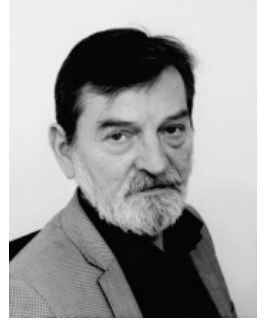

Eng. Andrzej Szmigiel is a researcher at the Department of Transport Warsaw University of Technology in the Department of Telecommunications Transport. Its main scope of the research are parking systems, transmission of information, quality information. It also deals with the analysis of information transmitted from parking systems (Share 10\%). 


\section{WPLYW NIEZAWODNOŚCI SYSTEMÓW TELEINFORMATYCZNYCH NA JAKOŚĆ PRZESYLANYCH INFORMACJI}

\section{Wstęp}

W pracy opisano wpływ niezawodności na jakoś informacji IQ (ang. - Information Quality) przesyłanych w systemach teleinformatycznych. Wg [2] jedną ze składowych IQ jest niezawodność obok takich właściwości jak relatywność, dokładność, aktualność, kompletność, spójność, odpowiedniość, dostępność, wiarygodność, przystawalność. Każda $\mathrm{z}$ tych składowych IQ jest niezależna $\mathrm{i}$ aby poprawnie oszacować wartość IQ należy użyć jednej z metod modelowanie niepewności. W artykule tym zastosowano metodę hybrydową opracowaną wspólnie przez jednego z autorów artykułu przedstawioną w [8]. Metoda ta opiera się na ewidencji matematycznej znanej jako teoria Dempstera-Shafera (DS) $[1,5]$ oraz szeregowych powiązaniach zależnych [4] nazwanych hybrydowymi IQ (hyb) [8]. W artykule tym przedstawiono sposób wyznaczania jakości informacji z wykorzystaniem metody hybrydowej. Model i obliczania ograniczono do wpływu niezawodności urządzeń oraz poprawności transmisji danych na tę jakość informacji. Wyznaczając IQ można w prosty sposób wykonać ocenę porównawczą systemów teleinformatycznych stosowanych $\mathrm{np}$. do nadzoru video (np: takie przedstawione $\mathrm{w}[3,6])$.

\section{Model jakości informacji dla wybranego fragmentu sieci teleinformatycznej}

Zaproponowano model jakości informacji dla fragmentu sieci teleinformatycznej przedstawionej i wyróżniony na rys. 1 .

Zależność jakości informacji od wielu właściwości systemu jest niezależna i równoległa. Jednak w niektórych przypadkach może się okazać, że należy uzależnić od kolejnych etapów wyznaczania tych równoległych właściwość. Z tego powodu wybrano metodę hybrydową, która zapewnia możliwość kalkulacji szeregowych i równoległych zależności (zależnych i niezależnych).

W przypadku struktury fragmentu sieci wybranego do modelowania można wyznaczyć jedną główną hipotezę h, której współczynnik będzie współczynnikiem jakości informacji IQ (hyb), oraz trzy hipotezy pośrednie dla każdego z zastosowanych urządzeń.

Hybrydowy model IQ bazujący na modelowaniu hybrydowym będzie opisany hipotezami pośrednimi:

h1 - poprawnie przekazano dane przez wybrany fragment sieci,

$\mathrm{h} 2$ - nastąpiły błędy transmisji wynikające $\mathrm{z}$ wad oprogramowania lub ingerencji zewnętrznej,

h3 - niepoprawna transmisja z powodu uszkodzenia urządzeń lub kabli. 
The impact of the reliability of teleinformation systems on the quality... Wpływ niezawodności systemów teleinformatycznych na jakość przesyłanych...

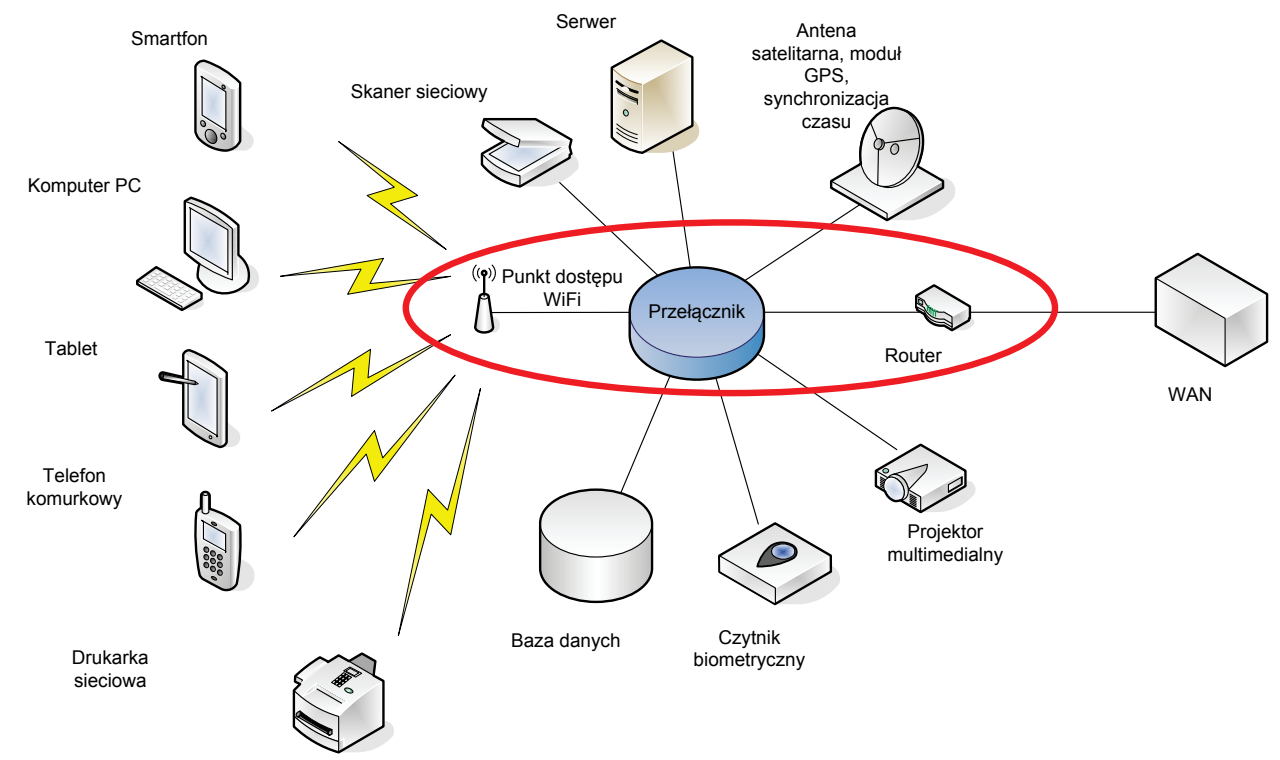

Rys. 1. Przykladowa sieć teleinformatyczna z zaznaczonym fragmentem użytym do modelowania jakości informacji. Źródło: opracowanie własne.

Obserwacje dla każdego z poszczególnych hipotez pośrednich będą następujące:

Dla hipotezy h1:

e1.1 - poprawnie przekazano dane przez punkt dostępu,

e1.2 - poprawnie przekazano dane przez przełącznik,

e1.3 - poprawnie przekazano dane przez router.

Dla hipotezy h2:

e2.1 - błąd transmisji lub wada oprogramowania punktu dostępowego,

e2.2 - błąd transmisji lub wada oprogramowania przełącznika,

e2.3 - błąd transmisji lub wada oprogramowania routera.

Dla hipotezy h3:

e3.1 - uszkodzenie punktu dostępowego,

e3.2 - uszkodzenie kabla,

e3.3 - uszkodzenie przełącznika,

e3.4 - uszkodzenie routera.

Wartości współczynników obserwacji zostały przedstawione w tabeli 1. Wartości te nie są empiryczne i służą tylko do zademonstrowania możliwości metody.

Tabela 1: Wartości współczynników obserwacji dla hipotez

pośrednich.
\begin{tabular}{|c|c|c|}
\hline $\mathrm{h} 1$ & $\mathrm{~h} 2$ & $\mathrm{~h} 3$ \\
\hline $\mathrm{e} 1.1=0,997$ & $\mathrm{e} 2.1=0,1 * 10^{-3}$ & $\mathrm{e} 3.1=0,1 * 10^{-3}$ \\
\hline $\mathrm{e} 1.2=0,999$ & $\mathrm{e} 2.2=0,5 * 10^{-4}$ & $\mathrm{e} 3.2=0,15^{*} 10^{-5}$ \\
\hline $\mathrm{e} 1.3=0,9992$ & $\mathrm{e} 2.3=0,3 * 10^{-3}$ & $\mathrm{e} 3.3=0,5 * 10^{-4}$ \\
\hline $\mathrm{x}$ & $\mathrm{x}$ & $\mathrm{e} 3.4=0,2 * 10^{-3}$ \\
\hline
\end{tabular}


Marek Stawowy, Zbigniew Kasprzyk, Andrzej Szmigiel

Graf zależnego modelu hipotez pośrednich modelu niezależnego przedstawia rys. 2. Na rys. 3 zostały umieszczone grafy poszczególnych, zależnych modeli dla każdej z hipotez pośrednich.

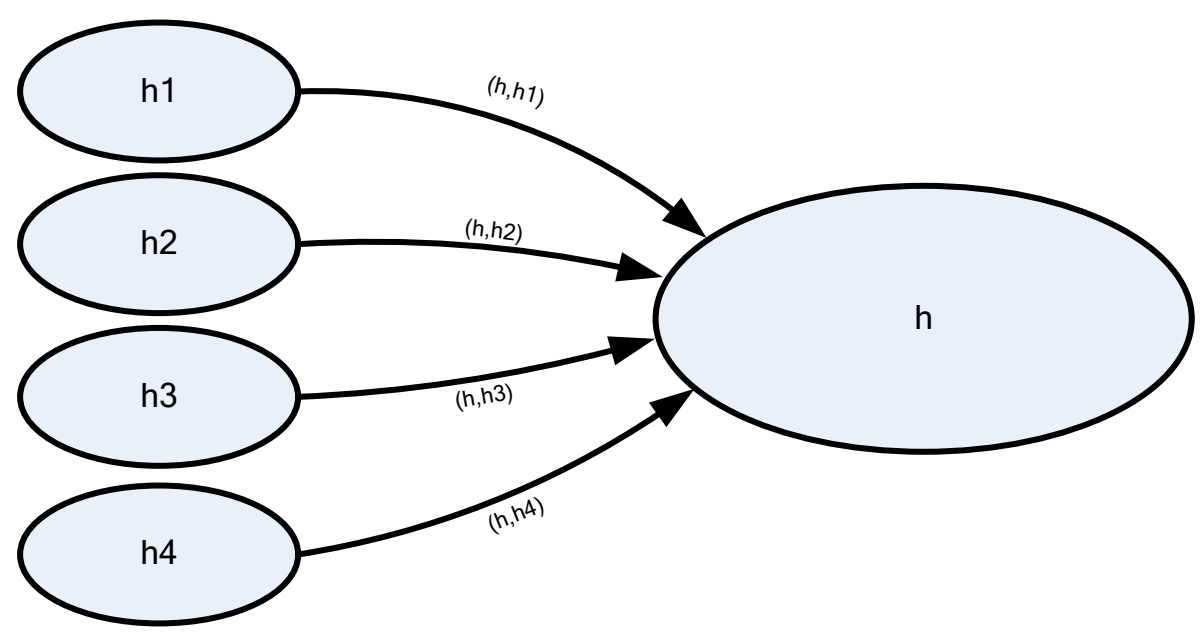

Rys. 2.Graf niezależnych hipotez pośrednich. Źródto: opracowanie własne.

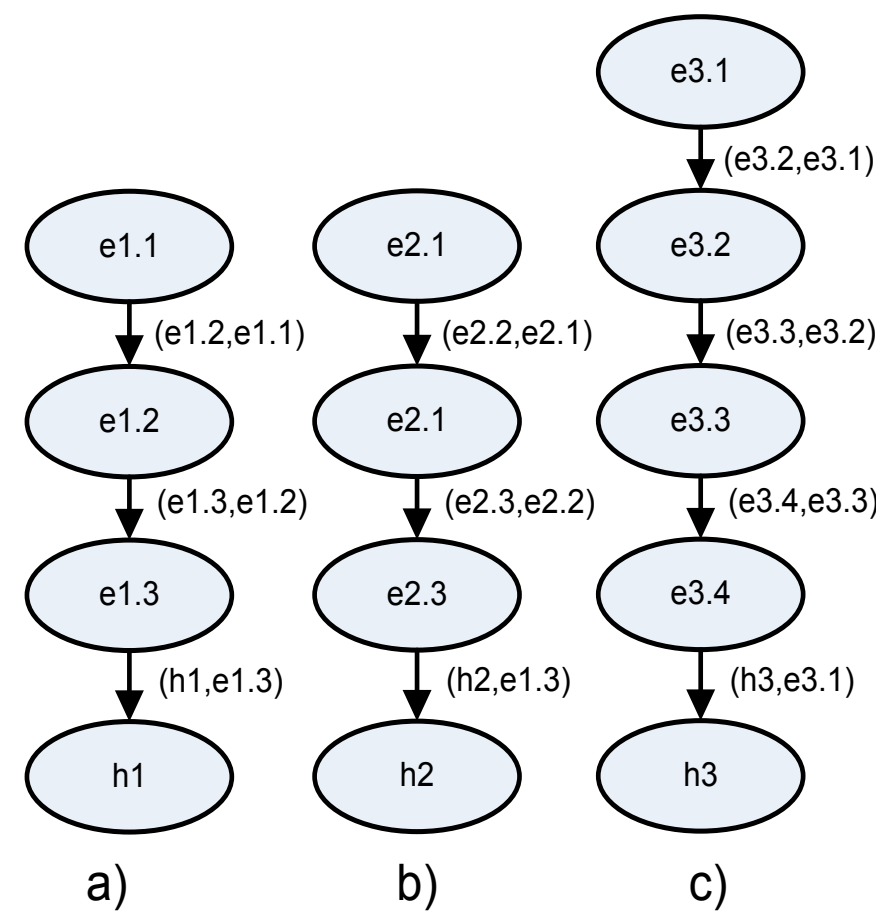

Rys. 3. Grafy zależnych obserwacji hipotez pośrednich a) h1, b) h2, c) h3. Źródto: opracowanie własne. 
The impact of the reliability of teleinformation systems on the quality...

Wplyw niezawodności systemów teleinformatycznych na jakość przesytanych...

Do wyznaczenia współczynników hipotez pośrednich wykorzystany iloczyn skalarny jak to opisano w [3,7]. W wyniku obliczeń otrzymano współczynniki przedstawione w tabeli 2 .

Tabela 2: Wartości wyznaczonych wspótczynników
hipotez pośrednich.
\begin{tabular}{|c|c|c|}
\hline $\mathrm{h} 1$ & $\mathrm{~h} 2$ & $\mathrm{~h} 3$ \\
\hline 0,988034976 & $0,15^{*} 10^{-11}$ & $0,15^{*} 10^{-17}$ \\
\hline
\end{tabular}

Dzięki teorii ewidencji matematycznej stworzonej przez Dempstera i Shafera (DS) [1,5] można syntezować właściwości $\mathrm{z}$ różnych źródeł niezależnych $[1,5,7,8]$. Wykorzystując tę teorię można wyznaczyć współczynnik hipotezy końcowej h w sposób opisany poniżej.

Zdefiniowane zostały trzy obserwacje skutków na podstawie obserwacji przyczyn i hipotez pośrednich:

Ze1 - dane poprawne, (h1),

Ze2 - dane nie dotarły z powodu błędów w oprogramowaniu lub ingerencji zewnętrznej (h2),

Ze3 - dane nie dotarły z powodu awarii sprzętowej (h3).

W tabelach 3, 4 i 5 przedstawione zostały kolejne etapy analizy obserwacji skutków.

$$
\begin{gathered}
\Theta=\{\mathrm{h} 1, \mathrm{~h} 2, \mathrm{~h} 3\} \\
\mathrm{m}_{1}(\Theta)=1 \\
\mathrm{~m}_{2}(\{\mathrm{~h} 1\})=0,988034976 \\
\mathrm{~m}_{2}(\Theta)=\mathrm{m}_{1}(\Theta)-\mathrm{m}_{2}(\{\mathrm{~h} 1\})
\end{gathered}
$$

Tabela 3: Obserwacja Ze1

\begin{tabular}{|c|c|c|}
\hline & $\mathrm{m}_{2}(\{\mathrm{~h} 1\})$ & $\mathrm{m}_{2}(\Theta)$ \\
\hline $\mathrm{m}_{1}(\Theta)$ & $\mathrm{m}_{3}(\{\mathrm{~h} 1\})$ & $\mathrm{m}_{3}(\Theta)$ \\
\hline \multicolumn{3}{|c|}{$\mathrm{m}_{4}(\{\mathrm{~h} 2\})=0,15 * 10^{-11}$} \\
$\mathrm{~m}_{4}(\Theta)=\mathrm{m}_{1}(\Theta)-\mathrm{m}_{4}(\{\mathrm{~h} 2\})$
\end{tabular}

Tabela 4: Obserwacja Ze2.

\begin{tabular}{|l|l|l|}
\hline & $\mathrm{m}_{4}(\{\mathrm{~h} 2\})$ & $\mathrm{m}_{4}(\Theta)$ \\
\hline $\mathrm{m}_{3}(\{\mathrm{~h} 1\})$ & $\mathrm{m}_{5}(\{\varnothing\})$ & $\mathrm{m}_{5}(\{\mathrm{~h} 1\})$ \\
\hline $\mathrm{m}_{3}(\Theta)$ & $\mathrm{m}_{5}(\{\mathrm{~h} 2\})$ & $\mathrm{m}_{5}(\Theta)$ \\
\hline
\end{tabular}

$$
\begin{gathered}
\mathrm{m}_{6}(\{\mathrm{~h} 3\})=0,15^{*} 10^{-17} \\
\mathrm{~m}_{6}(\Theta)=\mathrm{m}_{1}(\Theta)-\mathrm{m}_{6}(\{\mathrm{~h} 3\})
\end{gathered}
$$

Tabela 5: Obserwacja Ze3.

\begin{tabular}{|l|l|l|}
\hline & $\mathrm{m}_{6}(\{\mathrm{~h} 3\})$ & $\mathrm{m}_{6}(\Theta)$ \\
\hline $\mathrm{m}_{5}(\{\varnothing\})$ & $\mathrm{m}_{7}(\{\varnothing\})$ & $\mathrm{m}_{7}(\{\varnothing\})$ \\
\hline $\mathrm{m}_{5}(\{\mathrm{~h} 2\})$ & $\mathrm{m}_{7}(\{\mathrm{~h} 2\})$ & $\mathrm{m}_{7}(\{\mathrm{~h} 2\})$ \\
\hline $\mathrm{m}_{5}(\{\mathrm{~h} 1\})$ & $\mathrm{m}_{7}(\{\varnothing\})$ & $\mathrm{m}_{7}(\{\mathrm{~h} 1\})$ \\
\hline $\mathrm{m}_{5}(\Theta)$ & $\mathrm{m}_{7}(\{\mathrm{~h} 3\})$ & $\mathrm{m}_{7}(\Theta)$ \\
\hline
\end{tabular}

$$
\operatorname{Bel}(\mathrm{h} 1)=\mathrm{m}_{7}(\mathrm{~h} 1)
$$


$\mathrm{Na}$ podstawie rozważań w $[7,8]$ można złożyć, że Bel(h1) jest równe współczynnikowi jakości informacji przedstawionego wyżej modelu. Czyli $\mathrm{IQ}(\mathrm{hyb})=\operatorname{Bel}(\mathrm{h} 1)$ i po podstawieniu przykładowych wartości z tabeli 2 wynosi 0,988034975999982 .

\section{Symulacja i jej wyniki}

W symulacji komputerowej wykorzystano rozkłady normalne do przedstawienia wpływu niezawodności na jakość informacji. Wykonano 10000 kroków symulacji $\mathrm{z}$ odrzuceniem wartości spoza przemiału $<0,1>$. Program symulacyjny został napisany przez jednego $\mathrm{z}$ autorów na potrzeby tego artykułu.

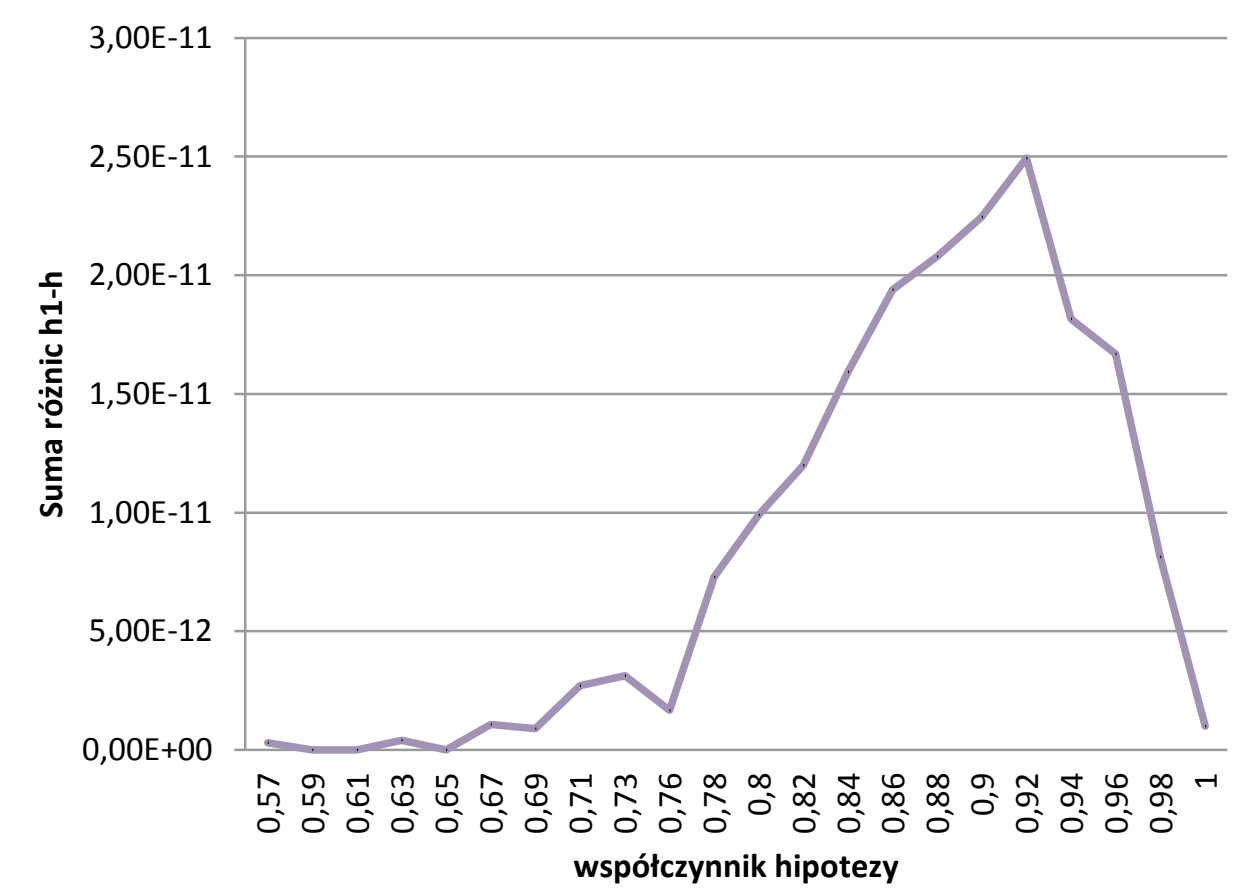

Rys. 4. Wynik symulacji-sumy różnic hl-h. Źródto: opracowanie własne.

Rys. 4 przedstawia wykres sum różnic hl-h w kolejnych krokach symulacji. Przebieg wykresu pokazuje jaki wielki wpływ na wartość współczynnika hipotezy h wpływ mają obserwacje pośrednie h2 i h3, czyli te związane z niezawodnością Rys. 5 przedstawia wyniki symulacji szacowania IQ(hyb). Wartość średnia odbiega nieznacznie od wartości wyliczonej w poprzednim rozdziale ze względu na to, że w symulacji były odrzucane wartości większe od 1 . 
The impact of the reliability of teleinformation systems on the quality... Wpływ niezawodności systemów teleinformatycznych na jakość przesyłanych...

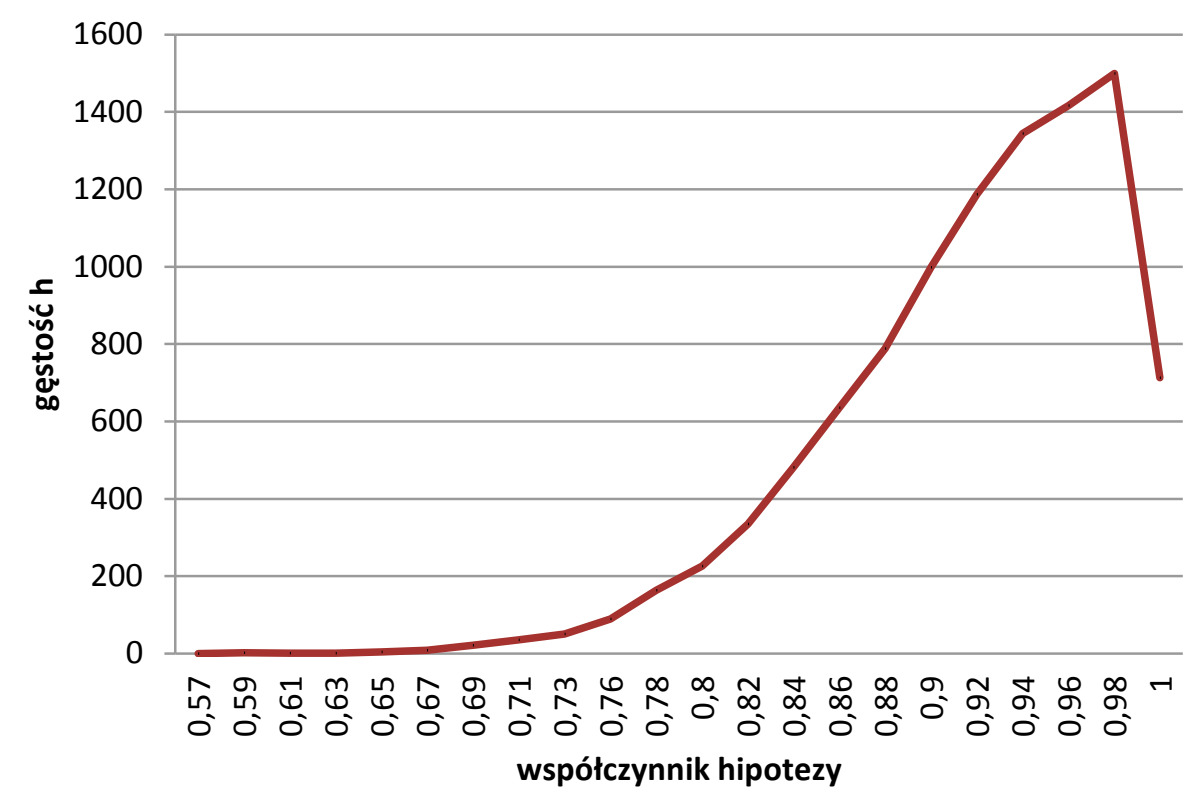

Rys. 5. Wynik symulacji-gęstość h. Źródto: opracowanie własne.

\section{Wnioski}

W artykule przedstawiono i wyznaczono oraz zasymulowana wpływ niezawodności na jakość informacji dla wybranego systemu teleinforamtycznego. Przedstawiona $\mathrm{w}$ artykule metoda, oparta na wyznaczaniu IQ poprzez modelowanie hybrydowe daje możliwość łatwego i szybkiego obliczenia jakości informacji na podstawie właściwości zależnych i niezależnych oraz szeregowych i równoległych. Jest także łatwa $\mathrm{w}$ implementacji jako program komputerowy poprzez powtarzalność większości obliczeń. W przypadku prezentowanym w artykule, metoda umożliwia szacowanie wpływu różnych właściwości na jakość informacji a w szczególności wpływ niezawodności. W kolejnym etapie należałoby uzależnić obliczania od czasu.

\section{Bibliografia}

[1] Dempster A. P. Upper and lower probabilities inducted by a multi-valued mapping. The Annals of Mathematical Statistics 38, 1967, 325-339. USA.

[2] Olaisen, J. Information quality factors and the cognitive authority of electronic information. Wormell, I. (Ed.), Information Quality: definitions and dimensions, London 1990: Taylor Graham.

[3] Lubkowski, P. and Laskowski, D. (2014) The selected issues of reliable identification of object in transport systems using video monitoring services. In the monograph Telematics - support for transport, given as the 
monographic publishing series - „Communications in Computer and Information Science”, Vol. 471. Springer-Verlag, Berlin Heidelberg, pp. 59-68.

[4] Pieczynski, W. Unsupervised Dempster-Shafer fusion of dependent sensors, Proc. 4th IEEE Southwest Symp. Image Analysis and Interpretation, 2000.

[5] Shafer, G. A mathematical theory of evidence. Princeton University Press. Princeton 1976.

[6] Siergiejczyk M., Paś J., Rosiński A.: Evaluation of safety of highway CCTV system's maintenance process. The monograph „Telematics - support for transport”, editors: Mikulski J., given as the monographic publishing series „Communications in Computer and Information Science”, Vol. 471. SpringerVerlag, Berlin Heidelberg 2014. pp. 69-79.

[7] Stawowy M., Comparison of Uncertainty Models of Impact of Teleinformation Devices Reliability on Information Quality, Proceedings of the European Safety and Reliability Conference ESREL 2014", editors Nowakowski T., Młyńczak M., Jodejko-Pietruczuk A. \& WerbińskaWojciechowska S. The publisher: CRC Press/Balkema, 2015. Pages 2329-2333.

[8] Stawowy M., Dziula P., Comparison of uncertainty multilayer models of impact of teleinformation devices reliability on Information Quality, Proceedings of the European Safety and Reliability Conference ESREL 2015, editors Luca Podofillini,Bruno Sudret,Bozidar Stojadinovic,Enrico Zio,Wolfgang Kröger CRC Press/Balkema, 2015. pp 2685-2691.

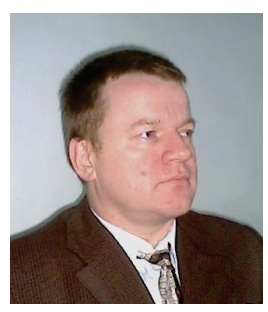

Marek Stawowy - Adiunkt wydzialu Transportu Politechniki Warszawskiej. Zainteresowania naukowe: komputerowa analiza obrazów, jakość informacji, modelowanie niepewności jakości informacji. Autor kilkudziesięciu artykutów naukowotechnicznych.

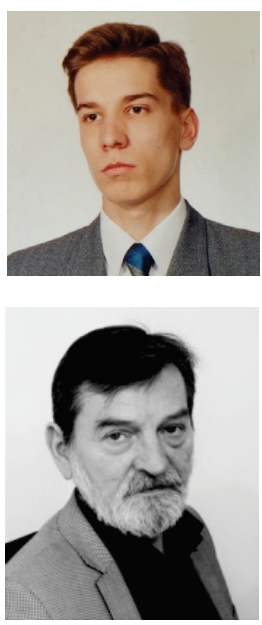

Zbigniew Kasprzyk - Adiunkt wydzialu Transportu Politechniki Warszawskiej. Zainteresowania naukowe: modelowanie systemów poboru opłat, modelowanie funkcji urządzeń telematyki transportu, analiza eksploatacyjna systemów światlowodowych torów telekomunikacyjnych (pomiary $i$ modernizacja), analiza niezawodnościowa rozwiazań sieci teleinformatycznych środków transportu. Autor kilkudziesięciu artykułów naukowo-technicznych.

Inz. Andrzej Szmigiel jest pracownikiem naukowo-badawczym na Wydziale Transportu Politechniki Warszawskiej w Zaktadzie Telekomunikacji w Transporcie. Jego głównym zakresem badawczym sa systemy parkingowe, przesytanie informacji, jakość informacji. Zajmuje się również analiza informacji przesytanych z systemów parkingowych. 
The impact of the reliability of teleinformation systems on the quality...

Wpływ niezawodności systemów teleinformatycznych na jakość przesyłanych... 